\title{
Photoreceptor Layer Thinning over Drusen in Eyes with Age-Related Macular Degeneration Imaged In Vivo with Spectral- Domain Optical Coherence Tomography
}

Stefanie G. Schuman, MD, ${ }^{1}$ Anjum F. Koreishi, BS, ${ }^{1}$ Sina Farsiu, PhD, ${ }^{1}$ Sin-ho Jung, PhD,${ }^{2}$ Joseph A. Izatt, PhD, ${ }^{1,3}$ Cynthia A. Toth, $\mathrm{MD}^{1,3}$

Purpose: Detect changes in the neurosensory retina using spectral-domain optical coherence tomography (SD OCT) imaging over drusen in age-related macular degeneration (AMD). Quantitative imaging biomarkers may aid in defining risk of disease progression.

Design: Cross-sectional, case-control study evaluating SD OCT testing in AMD.

Participants and Controls: Seventeen eyes of 12 subjects with nonneovascular AMD and drusen and 17 eyes of 10 age-matched control subjects.

Methods: Spectral-domain OCT imaging across the fovea in the study eye with multiple 10- to 12-mm scans of 1000 A scans each.

Main Outcome Measures: In summed SD OCT scans, the height of individual retinal layers either over drusen or at corresponding locations in the control eye and qualitative changes in retinal layers over drusen. Secondary measures included photoreceptor layer (PRL) area, inner retinal area, and retinal pigment epithelium (RPE)/drusen area.

Results: The PRL was thinned over $97 \%$ of drusen, average PRL thickness was reduced by $27.5 \%$ over drusen compared with over a similar location in controls, and the finding of a difference was valid and significant $(P=0.004)$. Photoreceptor outer segments were absent over at least 1 druse in $47 \%$ of eyes. Despite thinning of the PRL, inner retinal thickness remained unchanged. We observed 2 types of hyperreflective abnormalities in the neurosensory retina over drusen. Distinct hyperreflective speckled patterns occurred over drusen in $41 \%$ of AMD eyes and never in control eyes. A prominent hyperreflective haze was present in the photoreceptor nuclear layer over drusen in $67 \%$ of AMD eyes and more subtly in the photoreceptor nuclear layer in $18 \%$ of control eyes (no drusen).

Conclusions: With SD OCT as used in this study, we can easily detect and measure changes in PRL over drusen. Decreased PRL thickness over drusen suggests a degenerative process, with cell loss leading to decreased visual function. The hyperreflective foci overlying drusen are likely to represent progression of disease RPE cell migration into the retina and possible photoreceptor degeneration or glial scar formation. A longitudinal study using SD OCT to examine and measure the neurosensory retina over drusen will resolve the timeline of degenerative changes relative to druse formation.

Financial Disclosure(s): Proprietary or commercial disclosure may be found after the references. Ophthalmology 2009;116:488-496 @ 2009 by the American Academy of Ophthalmology.

Herein are reported the results from a search for imaging biomarkers of neurosensory retinal injury resulting from age-related macular degeneration (AMD), which is the leading cause of visual impairment in patients older than 65 years in the developed world. ${ }^{1,2}$ An estimated 8 million persons older than 55 years in the United States have monocular or binocular intermediate AMD or monocular advanced AMD. If neurosensory retinal injury is detected and measured promptly, then the recent advances in genetics, cell and molecular biology, and pharmacologic therapeutic approaches can be studied more effectively and applied to benefit AMD treatment. ${ }^{3,4}$
Drusen are extracellular deposits that accumulate between the retinal pigment epithelium (RPE) and the inner collagenous layer of Bruch's membrane ${ }^{5}$ and are known as a risk factor for the initial pathologic stage of AMD., 5 complement factor $\mathrm{H}(\mathrm{CFH})$ gene haplotypes have been associated with both the presence of drusen and advanced AMD. ${ }^{7}$ It is hypothesized that patients have a progressive loss of vision because of accumulation of drusen. ${ }^{2}$ There have been several postmortem studies of the relationship between photoreceptors and underlying drusen. Curcio et $\mathrm{al}^{8}$ reported a correlation between the presence of drusen and photoreceptor cell death in the retinas of eyes diag- 
nosed with AMD. Johnson et al ${ }^{9,10}$ identified both structural and functional photoreceptor changes in postmortem studies of retina over drusen. They documented focal changes in photoreceptor cell density, gene expression, and synapses over drusen. Other studies showed that lipid peroxidase in Bruch's membrane ${ }^{11}$ or RPE lysosomes ${ }^{12}$ causes RPE cell death, initiating the AMD pathologic process. It also has been proposed that the accumulation of abnormal material between the outer retina and the choriocapillaris interferes with the exchange of nutrients and waste products, inducing RPE or neural retinal damage, or both. ${ }^{13,14}$

Although appearance of a few drusen is normal with aging, greater accumulation of extracellular sub-RPE deposits is an indication of AMD, which later may progress to more severe forms such as choroidal neovascularization and nonneovascular geographic atrophy. Large multicenter studies have been undertaken to prevent or delay the onset of visual loss resulting from AMD. The Age-Related Eye Disease Study (AREDS) reported that AMD patients with advanced cases of dry AMD or vision loss resulting from wet AMD in 1 eye or extensive intermediate size drusen, at least 1 large druse, or noncentral geographic atrophy in both eyes could delay the onset of severe AMD and its accompanying vision loss by taking a high-dose vitamin and mineral supplement. ${ }^{15}$ Earlier identification of the patients at higher risk for developing advanced AMD allows even earlier protective intervention to reduce vision loss. ${ }^{16}$

The current measure of the level of early AMD is based on the size and extent of drusen, not on an objective measure of the neurosensory retina. ${ }^{17}$ Subjective testing of neurosensory retinal function, such as with the preferential hyperacuity perimeter, has been suggested as an indicator of the level of AMD; however, this testing requires patient interaction and feedback as an endpoint. ${ }^{18,19}$ Unfortunately, after severe AMD develops (either neovascular AMD or geographic atrophy), most treatments have suboptimal visual outcomes. Therefore, novel approaches to identify patients at high risk for developing severe AMD and to provide early protective intervention are critical for reducing vision loss resulting from this disease.

This study examined structural neurosensory retinal abnormalities in early AMD relative to sites of drusen. The purpose of this study was to investigate the effects of drusen on the overlying retina in vivo. The in vivo study of the photoreceptor layer (PRL) morphologic changes may relate closely to histopathologic studies carried out by Bressler et $\mathrm{al}^{20}$ and Green, ${ }^{21}$ which demonstrate a relationship between the loss of RPE and PRL. Unlike previous reports, which studied photoreceptor degeneration through histopathologic analysis, ${ }^{9,10}$ this study used in vivo high-resolution optical imaging. Although such imaging may be limited by relying on the relatively weak reflectivity contrast of intraretinal structures, it gains from the simplicity of noninvasive clinical testing and from the absence of postmortem artifacts that confound histopathologic studies.

\section{Patients and Methods}

Optical coherence tomography (OCT) is the only in vivo imaging method capable of resolving cross-sectional retinal substructure (Fig 1A). The novel spectral-domain optical coherence tomography (SD OCT) systems produce cross-sectional high-resolution images of tissue and achieve axial resolution of approximately 5 $\mu \mathrm{m}$ (Fig 1B). Spectral-domain OCT (20 $000 \mathrm{~A}$ scans/second) is 50 times faster than the conventional time-domain OCT (400 A scans/second, as in Stratus OCT; Carl Zeiss Meditec, Dublin, CA). Therefore, ophthalmic imaging via SD OCT significantly reduces artifacts because of patient movement and ocular contractions. ${ }^{22}$ Spectral-domain OCT is well suited to imaging the changes seen in AMD, because RPE, the site of many pathologic changes in AMD, is well delineated with this imaging. Differences in reflectivity between different intraretinal tissue layers and other material such as drusen allow identification of pathologic structures (Fig 1B). Moreover, in a fraction of the time required to capture a single time-domain OCT image, tens of SD OCT B scans (Fig 1B) can be recorded, registered, and averaged, creating an image with much less noise than any individual frame (Fig 1C). ${ }^{23}$

All subjects were enrolled in this Duke University Institutional Review Board-approved study between August 9, 2005, and January 26, 2007, and provided informed consent for SD OCT imaging and investigator access to ophthalmic records. Enrolled subjects were controls with no known ocular pathologic features, other than mild to moderate cataract, or those who had a clinical diagnosis of nonneovascular AMD on fundus examination in at least 1 eye, with at least 1 druse of more than $125 \mu \mathrm{m}$ in diameter. Eyes with scans of poor resolution of retinal layers or with geographic atrophy, choroidal neovascularization, or subretinal fluid were excluded.

The SD OCT system used in this study was an investigational device developed in the laboratory of Dr Joseph Izatt of the Duke University Department of Biomedical Engineering that was enhanced for macular imaging of AMD in collaboration with Cynthia Toth, MD. The system light source is a superluminescent diode developed by Superlum Diodes Ltd. (Moscow, Russia) with wavelength centered at $840 \mathrm{~nm}$ and bandwidth of $49 \mathrm{~nm}$. The power incident on the retina during SD OCT scanning is $500 \pm 50 \mu \mathrm{W}$ over a maximum of 6 seconds, well below the accepted American National Standards Institute (ANSI) extended exposure limit of $700 \mu \mathrm{W}$ over 8 hours. ${ }^{24}$ The incident light wavelength was $840 \mathrm{~nm}$ with 49-nm bandwidth. The axial resolution of the SD OCT system was approximately $4.5 \mu \mathrm{m}$, the transverse resolution was approximately $10 \mu \mathrm{m}$, and the acquisition time was set at $20000 \mathrm{~A}$ scan/second.

In each eye, regardless of the drusen density, $15 \mathrm{~B}$ scans per eye with $1000 \mathrm{~A}$ scans/B scan were acquired by scanning repeatedly across the center of the fovea (nasal to temporal) in approximately 0.75 seconds. Bioptigen SD OCT software (version 1.4; Bioptigen, Inc., Research Triangle Park, NC) was used for SD OCT scanning and image acquisition. The lateral dimensions of the scans were $12 \mathrm{~mm}\left(39^{\circ}\right)$ in $3 \mathrm{AMD}$ eyes and $10 \mathrm{~mm}\left(33^{\circ}\right)$ in 14 AMD eyes and 17 control eyes. All scans were scaled for proper comparison and analysis.

Stacks of 15 SD OCT B scans were imported to ImageJ (freeware; National Institutes of Health, Bethesda, MD) for analysis. In each stack, the shapes of the retinal and subretinal structures were inspected visually, such as the RPE and the vessel pattern. To reduce patient motion artifacts, the images with significantly different structures compared with most of the B scans in each stack were discarded. Sequential alignment of the remaining 8 to $15 \mathrm{~B}$ scans (registering frame no. 1 to frame no. 2 , and then frame no. 2 to frame no. 3 , and so forth) was accomplished by 

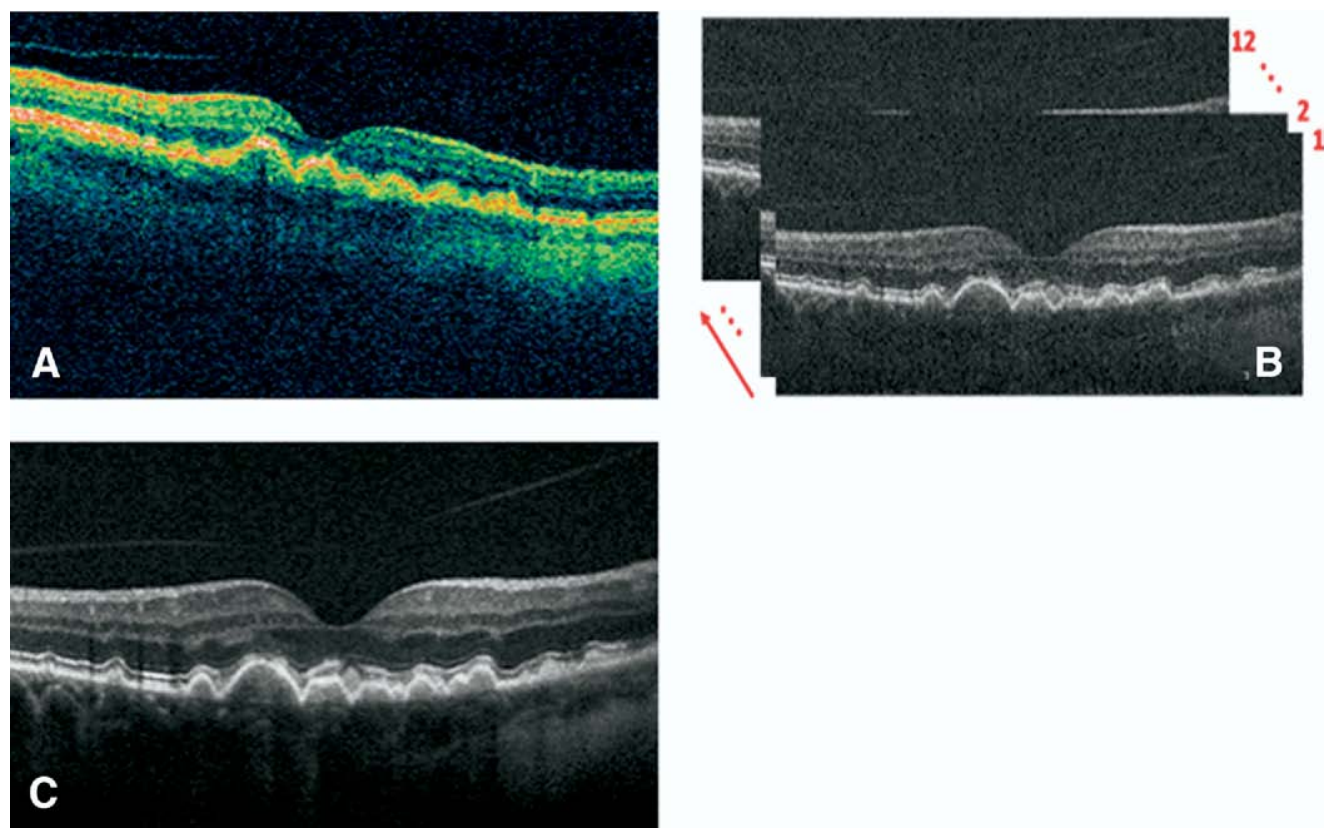

Figure 1. Examples of the high-quality spectral-domain optical coherence tomography (SD OCT) images of the macula in a patient with age-related macular degeneration used for this study compared with conventional time-domain OCT systems. A, Time-domain OCT image (Stratus, Carl Zeiss Meditec, Dublin, CA) in which drusen area appears fuzzy. B, Individual image (B scan) of the SD OCT system showing a significantly higher spatial resolution and better noise characteristics than images from the conventional time-domain OCT system. The image quality can be enhanced further by registering and fusing a sequence of captured B scans. Arrow shows direction of image fusion (averaging) C, Image created by registering and averaging 12 raw SD OCT B scans, with significantly higher signal-to-noise power ratio.
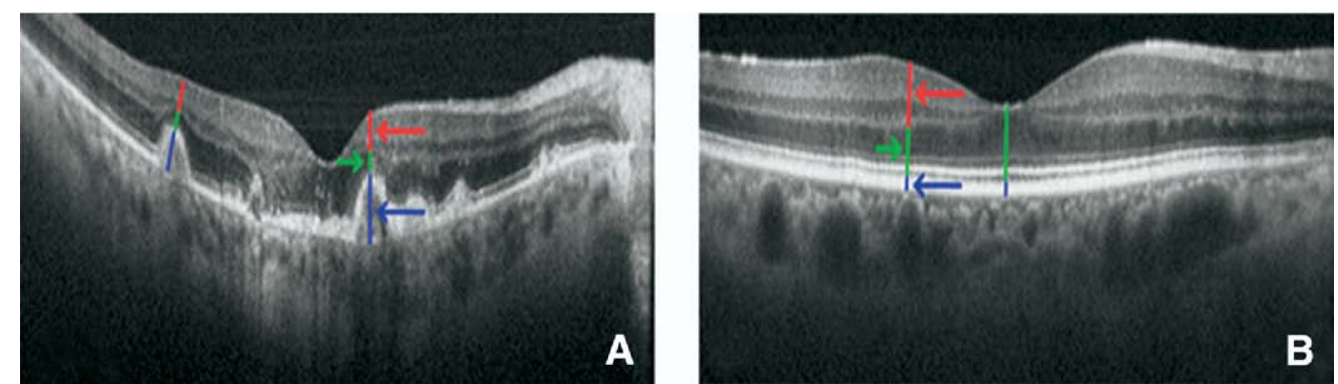

Figure 2. Images demonstrating measurement of the thickness of the retinal layers in eyes with age-related macular degeneration (AMD) and normal eyes. A, On each druse of the AMD eye, the druse height (including retinal pigment epithelium [RPE]), the photoreceptor layer (PRL) height, and the inner retina layer heights are marked with blue, green, and red lines (and arrows with matching colors), respectively. B, Normal eye using similar color scheme. Because of the absence of druse, the blue line represents the height of the RPE layer only in the control eye. Note the thinning of the PRL in (A) compared with (B).
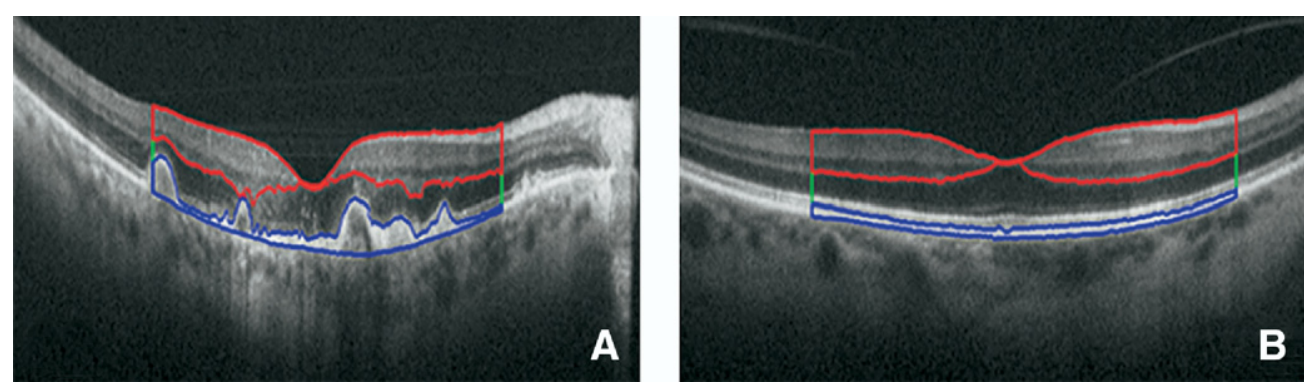

Figure 3. Images showing 3 distinct layer areas that were semiautomatically segmented using Amira software following the layer definitions in Figure 2: the segmented retinal pigment epithelium (RPE) and drusen (blue line), photoreceptor layer (green line), and inner retina (IR) layers (red line) in (A) a subject with age-related macular degeneration and (B) a control eye, respectively. Only the outer borders of the photoreceptor layer, which is located between the RPE and IR layers, are marked. 
using the StackReg plug-in (Biomedical Imaging Group, Swiss Federal Institute of Technology Lausanne, Lausanne, Switzerland) for ImageJ. ${ }^{25}$ These registered B scans then were averaged to reduce random background noise and to enhance visualization of retinal layers, creating a single high-quality B scan per eye. Figure $1 \mathrm{~B}, \mathrm{C}$ compares a set of unprocessed (raw) SD OCT B scans with a corresponding processed $\mathrm{B}$ scan.

The processed B scans were used for analysis in ImageJ. Qualitative scoring of AMD and control eyes included scoring for presence or absence of: cystoid macular edema, subretinal fluid, hyperreflective foci within the retina, and loss of any retinal layer, including photoreceptor inner or outer segments. The photoreceptor inner segments (located between the slightly reflective external limiting membrane and the more highly reflective band at the photoreceptor inner segment and outer segment junction) and outer segments (between the reflective band at the photoreceptor inner segment and outer segment junction and the RPE) represented a very thin layer. Therefore, these segments were scored as present or absent rather than with thickness measurements.

In each AMD eye, the width of each druse was measured. For each druse larger than $200 \mu \mathrm{m}$ across, the following measurements were made (Fig 2A): (1) height of druse, including RPE, which was the height measured from the base of the druse through the top of the RPE; (2) height of PRL, which was measured as the distance between the top of RPE and the outer plexiform layer (OPL); (3) height of inner retina (IR), which was measured as the distance between the OPL and internal limiting membrane (ILM). Retinal layers change in thickness across the macula. Therefore, in control eyes, height was measured (Fig 2B) at sites corresponding to the druse location, nasal or temporal to the fovea, in the AMD eyes. Because of the absence of drusen in these control eyes, for measurement 1 , only the height of the RPE was considered. The extent of PRL thinning over each druse was compared with the PRL height at the corresponding location in control eyes.

For layer segmentation and analysis, the summed B scans of both AMD and control eyes were imported into Amira software (version 4.1; Mercury Computer Systems, Carlsbad, CA). The 3 layers, delineated by the 3 height measurements explained above, were segmented semiautomatically with manual postcorrection (Fig 3). As explained in the next section, the areas of all layers were measured for comparison.

\section{Statistical Methods}

Although observations from different subjects are independent, those from the same eye and those from 2 eyes of each subject may be correlated. This type of data is called clustered data. Despite the possible dependency, the estimators obtained by ignoring dependency, called estimators based on a working independence model, are still unbiased or consistent for large numbers of independent subjects. The dependency was adjusted when the variance estimator of an estimator was calculated using the generalized estimating equation method of Liang and Zeger ${ }^{26}$; the 2 -sided $P$ values were reported when appropriate. Statistical methods are detailed further in Appendices 1, 2, 3, and 4.

\section{Results}

Seventeen eyes of 12 patients with nonneovascular AMD and 17 eyes of 10 control patients were scanned with SD OCT and underwent analysis. The age range of enrolled nonneovascular AMD subjects was 66 to 82 years, with a median age of 74 years; 6 patients and 9 eyes were female. The age range of enrolled control subjects was 62 to 74 years, with a median age of 68 years; 7 patients and 12 eyes were female. The mean visual acuity of the
AMD eyes was 0.22 logarithm of the minimum angle of resolution units (approximately 20/32-, with standard deviation of 0.21 ) and that of the control eyes was 0.06 logarithm of the minimum angle of resolution units (approximately 20/25+, with standard deviation of 0.14 ). Two control eyes had visual acuity of less than 20/40 because of cataract, with normal fundus examination results.

A qualitative difference in the PRL was visible focally over drusen. In most eyes, the drusen appeared to bulge into the PRL, without corresponding displacement of the overlying inner retinal layers. With SD OCT, evaluation of the PRL could be divided into photoreceptor nuclear layer, photoreceptor inner segments, and photoreceptor outer segments. In all control eyes, both the external limiting membrane (a faint reflective band above the photoreceptor inner segment and outer segment junction) and the photoreceptor outer segments (including the prominent hyperreflective band at the photoreceptor inner segment and outer segment junction) appeared grossly normal, and in many AMD eyes, these also appeared to be normal without thinning over the drusen. However, in $8(47 \%)$ of 17 AMD eyes, there was definite photoreceptor outer segment loss focally over at least 1 druse. In each case, this was also associated with disruption or loss of the reflective band at the photoreceptor inner segment and outer segment junction. It was difficult to judge whether the external limiting membrane was definitely abnormal at each of these sites. No cystoid macular edema, subretinal fluid, or loss of any inner retinal layer were found.

The photoreceptor nuclear layer showed faint hyperreflective haze in $3(18 \%)$ of 17 control eyes and prominent hyperreflective haze associated with sites of drusen in 11 (67\%) of 17 AMD eyes. The reflective haze in the AMD eyes was a focal, poorly demarcated increase in reflectivity of the photoreceptor nuclear layer over drusen or extending laterally on the nonfoveal side of the druse (Fig 4A,B). The haze was less common and less prominent over smaller drusen. It was not present over all large drusen and did not correlate with loss of the photoreceptor outer segments. Within the PRL, intense hyperreflective foci were present in 7 (41\%) of the 17 AMD eyes and in none of the control eyes. These foci were located over or laterally within $200 \mu \mathrm{m}$ of drusen and not in areas without drusen (Fig 5). Although the outer borders of the OPL demonstrated some blurring over drusen, otherwise there was no qualitative change to the inner retinal layers except for a shift in position over drusen.

Over almost every druse (97\% of cases), the PRL height (thickness) was decreased in the AMD eyes compared with the PRL height at the corresponding location in control eyes (Fig 6). There were only 2 sites (3\%) where the PRL height over drusen was greater than the corresponding PRL height in control eyes (the 2 points on the positive side of the "Change in the PRL Thickness (\%)" axis in Fig 6). As demonstrated in Appendix 1 (available at http://aaojournal.org), the claim that the PRL height was decreased in the AMD eyes compared with the PRL height at the corresponding location in control eyes was valid $(|\hat{T} / \hat{\sigma}|=2.87>0.95)$ and statistically significant $(P=0.004)$. The PRL was reduced an average of $27.5 \%$ over drusen in study AMD eyes compared with the corresponding retinal location in control eyes.

The decrease in photoreceptor thickness was more pronounced in areas over larger drusen compared with areas over smaller drusen. Drusen height had a much stronger correlation than drusen width with the extent of PRL thinning over drusen locations (Fig 7). As described in Appendix 2 (available at http://aaojournal.org), the slope of the linear regression line drusen height and width with percent PRL thinning were $-0.32(P=0.0355)$ and $-0.018(P=$ 0.420 ), respectively (Fig 7A,B).

Unlike PRL, the IR thickness remained almost unchanged over drusen. Figure 8 shows that in almost all distances from the fovea, the 
IR thickness in AMD eyes was very similar to a corresponding location relative to the fovea in the control eyes. As demonstrated in Appendix 1 (available at http://aaojournal.org), the claim that the IR height was decreased in the AMD eyes compared with the IR height at the corresponding location in control eyes was not valid $(|\hat{T} / \hat{\sigma}|=$ $0.6<0.95)$ and not statistically significant $(P=0.547)$.

Figure 9 compares the total area of the 3 retinal layers (PRL, inner retina, and RPE) measured in this report. Following Appendix 3 (available at http://aaojournal.org), the mean PRL area for AMD eyes was $1.06 \mathrm{~mm}^{2}$ (95\% confidence interval [CI], 1.011.11), compared with $1.10 \mathrm{~mm}^{2}$ (95\% CI, 1.06-1.13) in control eyes. This difference was not statistically significant $(P=0.153)$. The mean IR area in AMD eyes was $1.13 \mathrm{~mm}^{2}$ (95\% CI, 1.071.20), compared with $1.12 \mathrm{~mm}^{2}$ (95\% CI, 1.06-1.13) in control eyes; this difference was not statistically significant $(P=0.346)$. Finally, the mean RPE area in AMD eyes was $0.43 \mathrm{~mm}^{2}$ (95\% CI, $0.38-0.49)$, compared with $0.31 \mathrm{~mm}^{2}$ (95\% CI, 0.15-0.35) in control eyes; this difference was statistically significant $(P=$ $0.001)$. In $12(71 \%)$ of 17 AMD eyes, the RPE area was greater
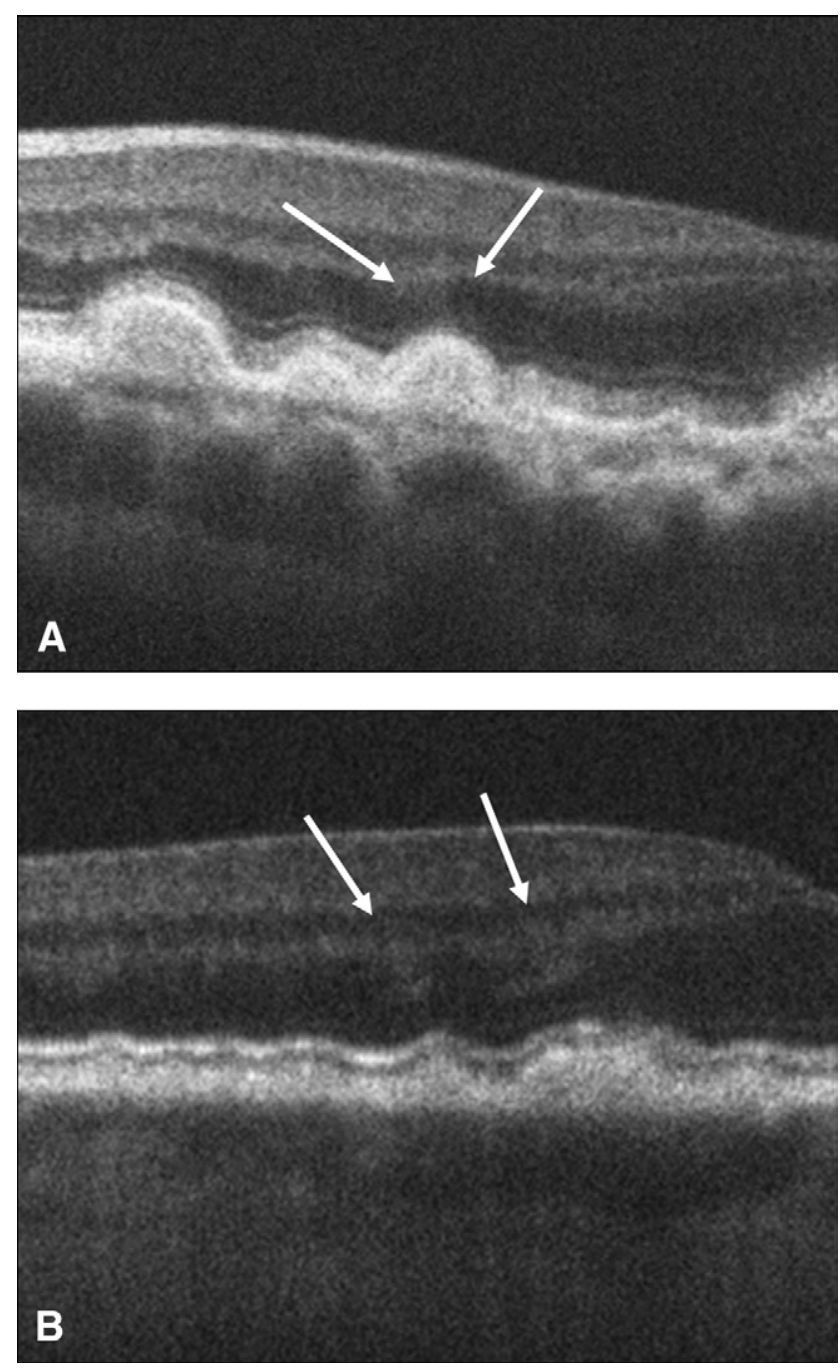

Figure 4. Spectral-domain optical coherence tomography (SD OCT) B scans ( $\mathbf{A}$ and $\mathbf{B}$ ) from 2 different eyes in which the arrows point to the sites of prominent diffuse hyperreflective haze located over drusen. In these eyes, the haze extends over the nonfoveal margin of the drusen. This haze was present over drusen in $67 \%$ of eyes.

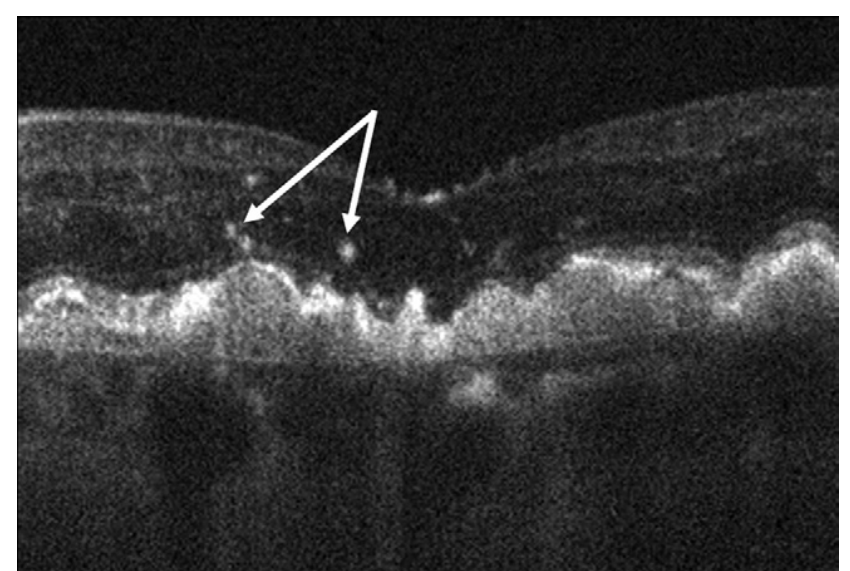

Figure 5. Spectral-domain optical coherence tomography B scan showing that focal hyperreflective speckling (arrows) was visible over and immediately adjacent to $41 \%$ of drusen and in none of the control images.

than the maximum area of any control eye. In the AMD eyes, the increase in the (total) RPE area (resulting from the presence of drusen) correlated with the decrease in the (total) PRL area ( $\hat{\rho}=$ $-0.65 P=0.05$ ), where, following Appendix 4 (available at http://aaojournal.org), $\hat{\rho}$ is the slope of the linear regression line. However in the control eyes, such correlation was small and without statistical significance $(\hat{\rho}=-0.02, P=0.46)$. In addition, the correlations between the PRL and IR areas in the AMD ( $\hat{\rho}=$ $-0.09, P=0.42)$ and the control $(\hat{\rho}=-0.09, P=0.41)$ eyes were not statistically significant. In the AMD eyes, the increase in the RPE area and the loss of the IR area showed a trend toward correlation $(\hat{\rho}=-0.49, P=0.14)$, whereas in control eyes, the increase in the RPE area was associated with an increase of the IR area $(\hat{\rho}=-0.52, P=0.008)$.

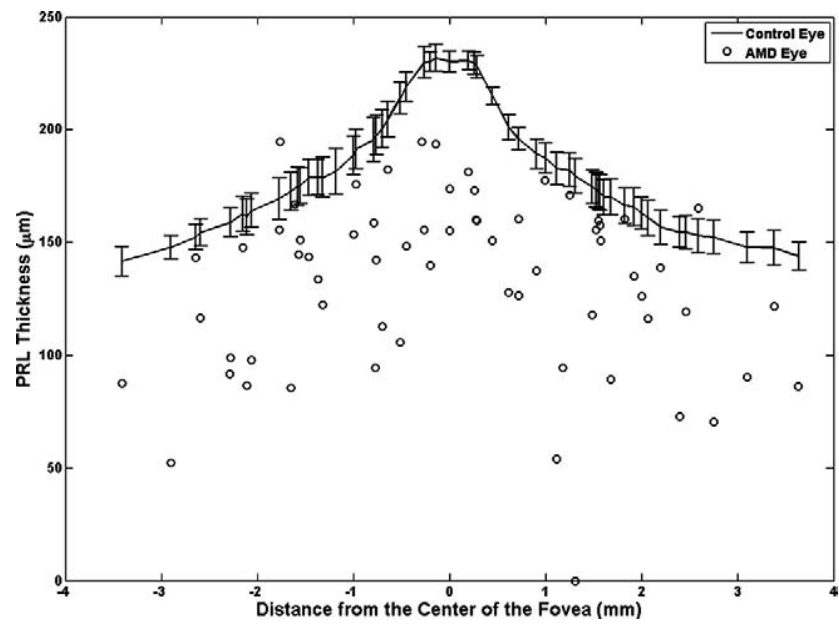

Figure 6. Graph showing the photoreceptor layer (PRL) height (thickness) at drusen locations in age-related macular degeneration (AMD) eyes compared with the corresponding values at similar distances from the center of the fovea in the control eyes. In almost all cases (97\%), the PRL thickness in the AMD eyes (circles) was thinner than that in the healthy control eyes (curved line with error bars representing the 95\% confidence interval). Nasal retina is to the left and temporal retina to the right of center of the graph. 


\section{Discussion}

Using high-speed high-resolution SD OCT imaging, retinal layers now can be measured in living eyes with nonneovascular AMD with a precision adequate to measure focal abnormalities over drusen. In the series of AMD eyes studied here, there was a significant and profound decrease in thickness of the PRL focally over drusen compared with that of age-matched control eyes, whereas the inner retinal layers were unaffected. The decrease in PRL correlated well with druse height and minimally with druse width. Although the difference in PRL area between AMD and control eyes was not significant (perhaps because of the very small area occupied by drusen relative to the total PRL area), the increase in RPE area (which included drusen) correlated with a decrease in PRL area in the AMD eyes. Despite the PRL changes over drusen, the mean area of the PRL layer did not differ between AMD and control eyes. This analysis supports an association between drusen and focal loss of the
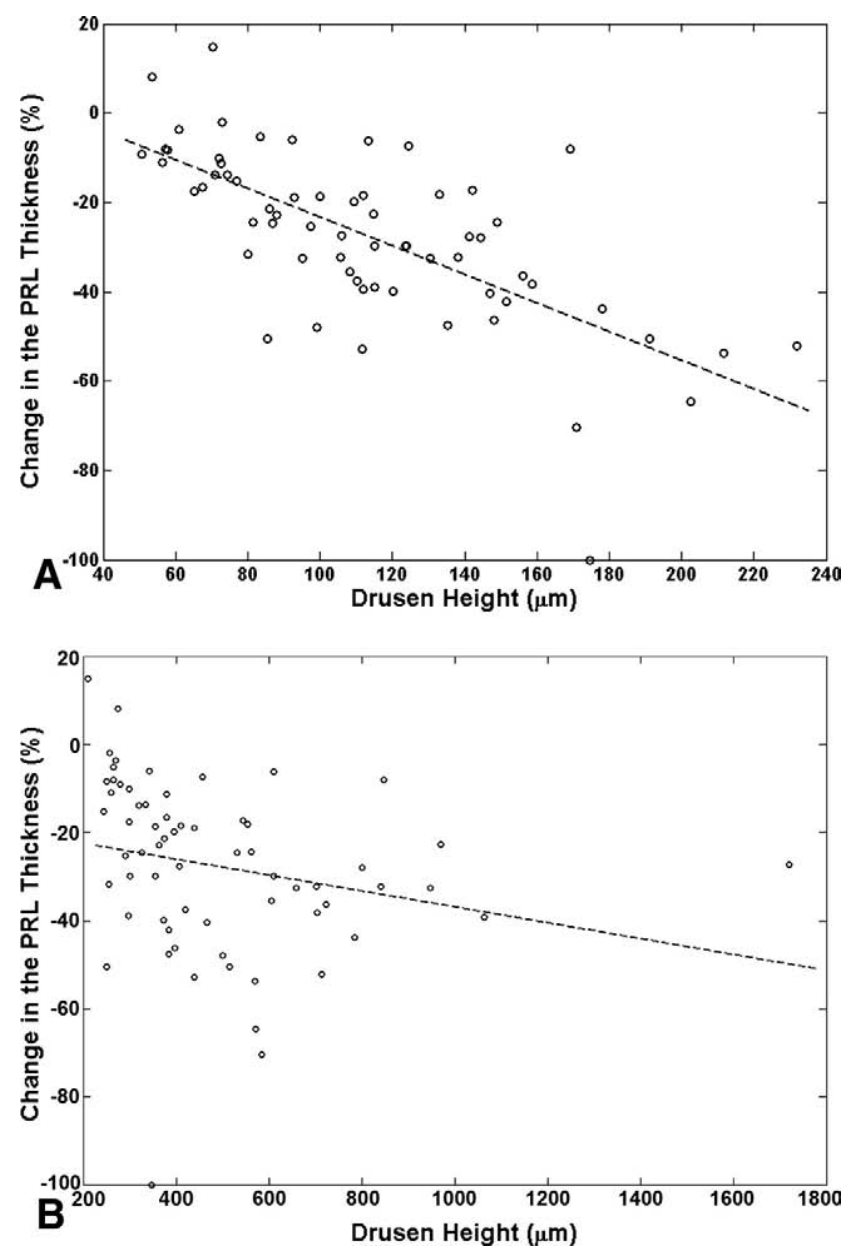

Figure 7. Scatterplots showing the relation between (A) drusen height and (B) drusen width with respect to the change in photoreceptor layer (PRL) thickness. The slope of the dashed line, which represents the linear regression fit (described in Appendix 2), is a measure of the correlation between the change in PRL thickness and drusen height (or width). It is evident from these scatterplots that the effect of the (A) drusen height in PRL thinning is more prominent than the effect of (B) drusen width.

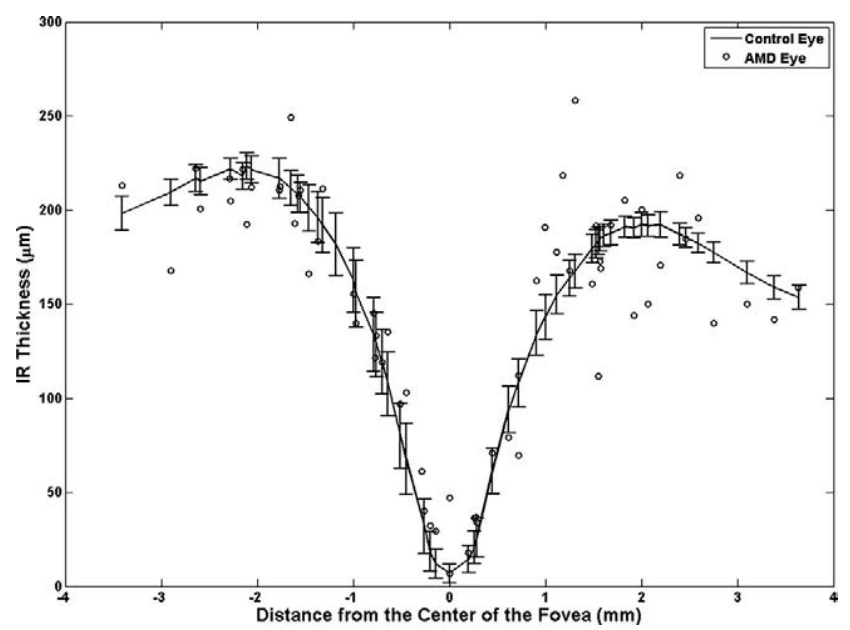

Figure 8. Graph showing the inner retinal (IR) height (thickness) measured at drusen locations in age-related macular degeneration (AMD) eyes compared with the corresponding values at similar distances from the center of the fovea in control eyes. The IR thickness in AMD eyes (circles) is not significantly different than that of the healthy control eyes (curved line with error bars representing the 95\% confidence interval). Nasal retina is to the left and temporal retina to the right of center of the graph.

photoreceptor layer in eyes with AMD, but suggests a lack of widespread photoreceptor loss (although there could be loss at a level not detectable in this small sample).

This finding of in vivo PRL thinning over drusen with preservation of the inner retinal layers correlates with previously published postmortem studies of photoreceptor loss in nonexudative AMD. Curcio et $\mathrm{al}^{8}$ and Curcio and Medeiros $^{27}$ identified moderate photoreceptor loss in the parafovea, with rod loss greater than cone loss at the same location. Focal loss of photoreceptors was not recorded over

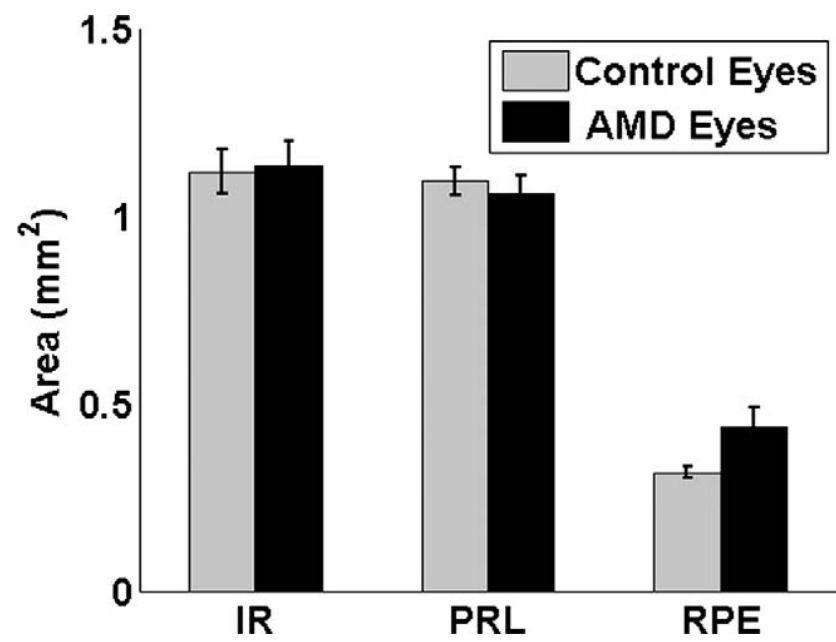

Figure 9. Bar graph showing the average measured total areas of the inner retinal (IR), photoreceptor layer (PRL), retinal pigment epithelial (RPE) layer in eyes of subjects with age-related macular degeneration (AMD) and control eyes. Error bars represent the $95 \%$ confidence interval. Aside from the evident change in the RPE area, no significant change is seen in the average area of IR and PRL. This further confirms the importance of the local thickness change study in Figure 6. 
specific drusen; however, the photoreceptor loss did seem to be more widespread than noted in this in vivo study. The postmortem finding of no difference in the ganglion cell layer between the nonexudative AMD eyes and controls agrees with the current finding of comparable inner retinal layer thickness between AMD and control eyes. The findings of decrease in PRL thickness by approximately $25 \%$ over drusen also compares favorably with that of Johnson et al, ${ }^{9}$ who reported a postmortem observation that photoreceptor density was consistently decreased (mean, approximately $30 \%$ ) over drusen. The authors divided drusen into 4 categories ranging from extremely small $(31-50 \mu \mathrm{m})$ to the largest of $91 \mu \mathrm{m}$ diameter and more. Although photoreceptor cell density progressively decreased as druse width increased in the postmortem study of smaller drusen, in this in vivo SD OCT study of eyes with drusen of more than 125 $\mu \mathrm{m}$, the photoreceptor layer thickness correlated closely to druse height and not as closely with druse width.

Note that the width of some of the drusen in this study clearly exceeded the typical maximum size on color fundus photographs of up to $434 \mu \mathrm{m}$ (based on the diameter of the I-2 ring and an optic nerve diameter of $1800 \mu \mathrm{m}$ ), after which it is more likely to be classified as drusenoid pigment epithelial detachment (there is no specific size end point between a druse, coalescent drusen, and drusenoid pigment epithelial detachment). In the AREDS grading system of AMD phenotypes, ${ }^{17}$ drusen are categorized by a hard or soft appearance based on "uniformity of density, sharpness of edges and thickness," 17 and maximum diameter also is recorded. Drusenoid pigment epithelial detachment is differentiated from drusen and scored as questionable, present, or absent, but size is not recorded. The differentiation between confluent drusen (margins in contact) and drusenoid PED is not defined explicitly in phenotyping studies, but becomes more obvious in cross-sectional imaging with SD OCT with lateral measurement of drusen. In the SD OCT images, there are multiple occurrences of drusen with confluent margins, resulting in a large lateral dimension for the drusen, although there is no single broad domed lesion (Fig 7B). Toth et al reported a greater lateral extent of drusen identified on SD OCT compared with corresponding color photographs for level 3 AMD eyes (Toth CA, Farsiu S, Chiu $\mathrm{SJ}$, et al. Automatic drusen segmentation and characterization in spectral domain optical coherence tomography [SD OCT] images of AMD eyes. Paper presented at: Association for Research in Vision and Ophthalmology Annual Meeting, May 1, 2008, Fort Lauderdale, Florida). In a separate publication, the authors are addressing a system of categorizing drusen by size, shape, and composition, so as to relate the SD OCT findings to conventional color fundus images. ${ }^{23}$

In addition to the quantitative changes in the retina over drusen, with high-speed high-resolution SD OCT imaging, we also can record and measure the qualitative difference in retinal imaging over drusen compared with that in agematched non-AMD control eyes. The notable qualitative changes on SD OCT were the absence of the photoreceptor outer segments with the associated loss or disruption of the highly reflective photoreceptor inner segment and outer segment junction, the hyperreflective haze within the remaining photoreceptor nuclear layer over drusen, and hyperreflective speckles over and adjacent to drusen. The findings of a decrease in photoreceptor height, focal loss of the photoreceptor outer segments, and abnormal reflectivity at the site of remaining photoreceptors over drusen supports the argument that neurosensory retinal degeneration is present in eyes with highrisk drusen and is present before the development of geographic atrophy or choroidal neovascularization.

The hyperreflective changes over drusen may represent multiple stages of a single process or potentially 2 different processes over drusen. Possible causes include an abnormal deposit such as pigment, displaced or migrated RPE cells or macrophages with pigment inside, exudates, lipid, or even blood within the retina or a degenerative retinal process. Some very intensely reflective intraretinal foci have been associated with hyperpigmentation on color fundus photographs. ${ }^{23}$ Focal pigment abnormalities have been associated with higher risk of progression to severe AMD, ${ }^{28-30}$ and hyperreflective sites on SD OCT may represent a precursor to larger pigment clumps, which are visible on fundus photographs, and thus may be an earlier risk indicator for druse progression. This question is being addressed in a longitudinal study ancillary to the AREDS 2. ${ }^{31}$ In the AREDS 2 Ancillary SD OCT Study (available online at http://clinicaltrails.gov/ct2/show/NCT00345176; accessed October 6, 2008), larger SD OCT image data sets across the entire macula are be compared with conventional imaging in highrisk drusen eyes with annual follow-up of up to 5 years.

An inflammatory process or a degenerative retinal process such as in synaptic terminals also may cause focal PRL hyperreflectivity if subcellular abnormalities increased the normal reflectivity of that site. The authors theorize that a degenerative cellular process may cause the less intense and more diffuse hyperreflective haze visible within the photoreceptor nuclear layer. Because SD OCT reflectivity changes range from mild and diffuse to more focal and intense, these sites may represent a spectrum of abnormalities in photoreceptors overlying drusen. In postmortem studies, Johnson et $\mathrm{al}^{9,10}$ found that photoreceptors overlying and flanking drusen exhibited morphologic signs of degeneration, including changes in localization of synaptic proteins within cells, the location and structure of the synaptic terminals, and in expression of stress response proteins. Although abnormalities in the synaptic terminal of photoreceptor cells also have been shown in an experimental model of neovascular AMD, from which $\mathrm{Ca}-$ icedo et $\mathrm{al}^{32}$ proposed that macrophages migrating into the neurosensory retina may initiate the neurosensory retinal dysfunction, a possible relationship between SD OCT hyperreflectivity in the PRL and synaptic terminal dysfunction requires further study for validation.

In this SD OCT study, images of the PRL over drusen were captured at a single time point in the progression of AMD. Fortunately, with this noncontact method of imaging the living eye, these measurements can be repeated over time to assess the relationships between drusen development and photoreceptor loss. To date, there are multiple theories regarding the relationship between drusen and the neurosensory retina in the early stages of AMD. These have ranged from photoreceptor degeneration (particularly rod loss) preceding further RPE loss and drusen progression ${ }^{8}$ to several theories of changes in Bruch's membrane, drusen 
formation, or RPE degeneration causing photoreceptor degeneration. ${ }^{9,33}$ For example, drusen may affect photoreceptors by physical displacement, which damages their structural integrity, or compromises the function of RPE cells, a prerequisite for normal photoreceptor cell function and maintenance of the retinal microenvironment. ${ }^{9}$ Drusen may block the normal diffusion of metabolic materials between the photoreceptors and choroidal blood supply, leading to concentration of waste ${ }^{13,14}$ near the RPE and inhibition of the diffusion of oxygen, glucose, and nutritive serumassociated molecules required to maintain the health of the outer retina and RPE. ${ }^{13,14}$ Although analysis of current SD OCT images will not aid in selecting between these theories, the use of these precise morphologic measurements over time, in conjunction with functional testing, should aid in the stratification of stages of disease progression. Moreover, because significant photoreceptor effects are already imaged with SD OCT in eyes with high-risk drusen, this imaging also is likely to be useful in investigating earlier events in drusen formation and photoreceptor change before high-risk drusen. Detection and analysis of change in photoreceptors over smaller drusen in longitudinal studies may result in earlier predictors of AMD progression. Alternately, because SD OCT enables detection of and measurement of changes in ultrastructural drusen components ${ }^{23}$ and the effect of drusen on the surrounding retina in vivo, it is appropriate to search for either a relationship between these events and progression to severe disease or earlier signs of choroidal neovascularization or of geographic atrophy development.

The focal qualitative and quantitative changes in the PRL over drusen suggest measurable photoreceptor loss and likely dysfunction. These may be useful biomarkers for visual impairment associated with drusen and may predict the subsequent course of disease progression. Because the anomalous findings varied notably from patient to patient, there is a possibility that they are associated with specific genotype, systemic biomarkers, or environmental exposure.

For SD OCT to be effective in AMD studies, researchers will need to know the reproducibility of qualitative grading, variance of drusen volume, photoreceptor, and other retinal layer measurements across a 3-dimensional region of interest in the macula. This is in contrast to the more limited sample across the macula in this study. Investigators in the aforementioned AREDS 2 Ancillary SD OCT Study will address these ideas in addition to the predictive value of qualitative and quantitative data in the longitudinal SD OCT imaging across a 3-dimensional central section of the macula.

AcknowledgmentsThe authors thank Aziz A. Khanifar, MD, for providing essential information for the database on which this study was built.

\section{References}

1. Klein R, Klein BE, Jensen SC, Meuer SM. The five-year incidence and progression of age-related maculopathy: the Beaver Dam Eye Study. Ophthalmology 1997;104:7-21.

2. Mitchell P, Smith W, Attebo K, Wang JJ. Prevalence of age-related maculopathy in Australia: the Blue Mountains Eye Study. Ophthalmology 1995;102:1450-60.
3. Rohrer B, Guo Y, Kunchithapautham K, Gilkeson GS. Eliminating complement factor D reduces photoreceptor susceptibility to light-induced damage. Invest Ophthalmol Vis Sci 2007;48:5282-9.

4. Wong RW, Richa DC, Hahn $\mathrm{P}$, et al. Iron toxicity as a potential factor in AMD. Retina 2007;27:997-1003.

5. Pauleikhoff D, Barondes MJ, Minassian D, et al. Drusen as risk factors in age-related macular disease. Am J Ophthalmol 1990;109:38-43.

6. Bressler SB, Maguire MG, Bressler NM, Fine SL, Macular Photocoagulation Study Group. Relationship of drusen and abnormalities of the retinal pigment epithelium to the prognosis of neovascular macular degeneration. Arch Ophthalmol 1990;108:1442-7.

7. Francis PJ, Schultz DW, Hamon S, et al. Haplotypes in the complement factor $\mathrm{H}(\mathrm{CFH})$ gene: associations with drusen and advanced age-related macular degeneration. PLoS ONE [serial online] 2007;2:e1197. Available at: http://www.plosone.org/ article/info:doi/10.1371/journal.pone.0001197. Accessed September 9, 2008.

8. Curcio CA, Medeiros NE, Millican CL. Photoreceptor loss in age-related macular degeneration. Invest Ophthalmol Vis Sci 1996;37:1236-49.

9. Johnson PT, Brown MN, Pulliam BC, et al. Synaptic pathology, altered gene expression, and degeneration in photoreceptors impacted by drusen. Invest Ophthalmol Vis Sci 2005;46: 4788-95.

10. Johnson PT, Lewis GP, Talaga KC, et al. Drusen-associated degeneration in the retina. Invest Ophthalmol Vis Sci 2003; 44:4481-8.

11. Spaide RF, Ho-Spaide WC, Browne RW, Armstrong D. Characterization of peroxidized lipids in Bruch's membrane. Retina 1999;19:141-7.

12. Kopitz J, Holz FG, Kaemmerer E, Schutt F. Lipids and lipid peroxidation products in the pathogenesis of age-related macular degeneration. Biochimie 2004;86:825-31.

13. Holz FG, Pauleikhoff D, Klein R, Bird AC. Pathogenesis of lesions in late age-related macular disease. Am J Ophthalmol 2004; 137:504-10.

14. Pauleikhoff D, Harper CA, Marshall J, Bird AC. Aging changes in Bruch's membrane: a histochemical and morphologic study. Ophthalmology 1990;97:171-8.

15. Age-Related Eye Disease Study Research Group. A randomized, placebo-controlled, clinical trial of high-dose supplementation with vitamins $\mathrm{C}$ and $\mathrm{E}$, beta carotene, and zinc for age-related macular degeneration and vision loss: AREDS report no. 8. Arch Ophthalmol 2001;119:1417-36.

16. Ross RJ, Verma V, Rosenberg KI, et al. Genetic markers and biomarkers for age-related macular degeneration. Expert Rev Ophthalmol 2007;2:443-57.

17. Age-Related Eye Disease Study Research Group. The AgeRelated Eye Disease Study system for classifying agerelated macular degeneration from stereoscopic color fundus photographs: the Age-Related Eye Disease Study report number 6. Am J Ophthalmol 2001;132:668-81.

18. Kampmeier J, Zorn MM, Lang GK, et al. Comparison of preferential hyperacuity perimeter (PHP) test and Amsler grid test in the diagnosis of different stages of age-related macular degeneration [in German]. Klin Monatsbl Augenheilkd 2006; 223:752-6.

19. Loewenstein A. The significance of early detection of agerelated macular: Richard \& Hinda Rosenthal Foundation Lecture, The Macula Society 29th Annual Meeting. Retina 2007; 27:873-8. 
20. Bressler NM, Silva JC, Bressler SB, et al. Clinicopathologic correlation of drusen and retinal pigment epithelial abnormalities in age-related macular degeneration. Retina 1994;14: $130-42$.

21. Green WR. Histopathology of age-related macular degeneration. Mol Vis [serial online] 1999;5:27. Available at: http://www. molvis.org.molvis.v5/a27/. Accessed September 9, 2008.

22. Chen TC, Cense B, Pierce MC, et al. Spectral domain optical coherence tomography: ultra-high speed, ultra-high resolution ophthalmic imaging. Arch Ophthalmol 2005; 123:1715-20.

23. Khanifar AA, Koreishi AF, Izatt JA, Toth CA. Drusen ultrastructure imaging with spectral domain optical coherence tomography in age-related macular degeneration. Ophthalmology 2008;115:1883-90.

24. American National Standard for the Safe Use of Lasers. ANSI Z136.1-2000. Orlando, FL: Laser Institute of America; 2000.

25. Thevenaz P, Ruttimann UE, Unser M. A pyramid approach to subpixel registration based on intensity. IEEE Trans Image Process 1998;7:27-41.

26. Liang KY, Zeger SL. Longitudinal data analysis using generalized linear models. Biometrika 1986;73:13-22.
27. Medeiros NE, Curcio CA. Preservation of ganglion cell layer neurons in age-related macular degeneration. Invest Ophthalmol Vis Sci 2001;42:795-803.

28. Buch H, Vinding $\mathrm{T}$, la Cour $\mathrm{M}$, et al. Risk factors for age-related maculopathy in a 14-year follow-up study: the Copenhagen City Eye Study. Acta Ophthalmol Scand 2005; 83:409-18.

29. Klein R, Klein BE, Knudtson MD, et al. Fifteen-year cumulative incidence of age-related macular degeneration: the Beaver Dam Eye Study. Ophthalmology 2007;114:253-62.

30. van Leeuwen R, Klaver CC, Vingerling JR, et al. The risk and natural course of age-related maculopathy: follow-up at $61 / 2$ years in the Rotterdam Study. Arch Ophthalmol 2003;121: $519-26$.

31. Coleman H, Chew E. Nutritional supplementation in age-related macular degeneration. Curr Opin Ophthalmol 2007;18:220-3.

32. Caicedo A, Espinosa-Heidmann DG, Hamasaki D, et al. Photoreceptor synapses degenerate early in experimental choroidal neovascularization. J Comp Neurol 2005;483:263-77.

33. Zarbin MA. Current concepts in the pathogenesis of agerelated macular degeneration. Arch Ophthalmol 2004;122: $598-614$.

\section{Footnotes and Financial Disclosures}

Originally received: February 23, 2008.

Final revision: October 7, 2008.

Accepted: October 7, 2008.

Available online: January 22, 2009.

Manuscript no. 2008-246.

${ }^{1}$ Duke University Medical Center, Department of Ophthalmology, Durham, North Carolina.

${ }^{2}$ Duke University Medical Center, Department of Biostatistics and Bioinformatics, Durham, North Carolina.

${ }^{3}$ Duke University Pratt School of Engineering, Department of Biomedical Engineering, Durham, North Carolina.

Financial Disclosure(s):

The author(s) have made the following disclosure(s):

Sina Farsiu - Research Support - North Carolina Biotechnology Center and Bioptigen Inc., Research Triangle Park, North Carolina.

Joseph A. Izatt - Investor - Bioptigen, Inc., Research Triangle Park, North Carolina.
Cynthia A. Toth - Research Support - Genentech, South San Francisco, California; Sirion Therapeutics, Tampa, Florida; Bioptigen, Inc., Research Triangle Park, North Carolina; Alcon Laboratories, Inc., Fort Worth, Texas; North Carolina Biotechnology Center, Research Triangle Park, North Carolina; Consultant - Genentech, South San Francisco, California; Patent Royalties - Alcon Laboratories, Inc., Fort Worth, Texas.

Supported in part by Bioptigen, Inc., Research Triangle Park, North Carolina; Duke University, Durham, North Carolina; and The North Carolina Biotechnology Center, Research Triangle Park, North Carolina; (Collaborative Funding Grant no.: 2007-CFG-8005); the National Institutes of Health, Bethesda, Maryland (grant nos.: R21 EY017393 and 1 UL1 RR024128-01 [S.-h.J.]).

Correspondence:

Cynthia A. Toth, MD, Duke University Eye Center, DUMC 3802, Durham NC 27710. E-mail: cynthia.toth@duke.edu. 


\section{Appendix 1. Comparison of the Photoreceptor Layer (or Inner Retina) Thickness over Drusen in Control Eyes versus Age-Related Macular Degeneration Eyes}

For the age-related macular degeneration (AMD) group, let $x_{l\left(i_{j} j_{l}\right)}$ denote the photoreceptor layer (PRL) thickness at location $l$ for eye $j_{l}$ of patient $i_{l}$ with a measurement at location (distance from fovea) $l$. Patient $i\left(=1, \ldots, n_{1}\right)$ contributes $m_{1 i}(=1$ or 2$)$ eyes, and $n_{1}=12$ patients with AMD contributed a total of $m_{1}=\sum_{i=1}^{n_{1}} m_{1 i}=17$ eyes. Each eye of an AMD patient has 1 to 12 drusen locations, which equals a total of $L_{1}=65$ drusen locations from 17 eyes.

Each control eye contributes $L_{2}=57$ observations, 1 for each of the 57 locations that are chosen exactly or very close to the $L_{1}=65$ drusen locations of the AMD group. For the control group, let $y_{l i j}$ denote the PRL thickness at location $l\left(=1, \ldots, L_{2}\right)$ for eye $j\left(=1, \ldots, m_{2 i}\right)$ of patient $i(=1, \ldots$, $n_{2}$ ). In the control group, $n_{2}=9$ healthy subjects contributed a total of $m_{2}=\sum_{i=1}^{n_{2}} m_{2 i}=17$ eyes.

\section{Testing}

For location $l$, we have one observation, $x_{l\left(i_{j} j_{l}\right)}$, from the AMD group and $L_{2}=17$ observations, $\left\{y_{l i^{\prime} j^{\prime}}, 1 \leq j^{\prime} \leq m_{2 i^{\prime}}\right.$, $\left.1 \leq i^{\prime} \leq n_{2}\right\}$ from the control group. Let $\hat{F}_{l}\left(x_{l\left(i_{l} j_{l}\right)}\right)=$ $m_{2}^{-1} \sum_{i^{2}=1}^{n_{2}} \sum_{j^{\prime}=1}^{m_{2 i}} I\left(y_{l i^{\prime} j^{\prime}}>x_{l\left(i_{j} j_{l}\right.}\right)$ denote the proportion of the control eyes whose PRL thickness is larger than that from a diseased eye at the same location $l$. We have $\hat{F}_{l}\left(x_{l\left(i_{l} j_{l}\right)}\right) \approx$ $1 / 2$ if the average PRL thickness is not changed by drusen, and $\hat{F}_{l}\left(x_{l\left(i_{l} j_{l}\right)}\right)$ will tend to be larger than $1 / 2$ if the average PRL thickness decreases by drusen. Let $D$ denote the range of distance. We consider following hypotheses

$$
\begin{aligned}
& H_{0}: x_{l\left(i_{l} j_{l}\right)} \text { and } y_{l i^{\prime} j^{\prime}} \text { are identically distributed for all } \\
& \quad l \in D
\end{aligned}
$$

against

$$
\begin{aligned}
& H_{1}: x_{l\left(i_{l} j_{l}\right)} \text { and } y_{l i^{\prime} j^{\prime}} \text { are differentially distributed for } \\
& \text { some } l \in D \text {. }
\end{aligned}
$$

Let $F_{l}(\cdot)$ denote the expected value of $\hat{F}_{l}(\cdot)$. Under $H_{0}$, the expected value of $F_{l}\left(x_{l\left(i_{l} j_{l}\right)}\right)$ is $1 / 2$ and $\hat{F}_{l}(\cdot)$ is a consistent estimator of $F_{l}(\cdot)$ for large $n_{2}$. So, we propose to reject $H_{0}$ if the absolute value of

$$
T=n_{1}^{-1 / 2} \sum_{l=1}^{L}\left\{\hat{F}_{l}\left(x_{l\left(i_{l} j_{l}\right)}\right)-1 / 2\right\}
$$

is large.

We derive the distribution of $T$ using the large sample theory (with respect to $n_{1}$ and $n_{2}$ ) for clustered data. ${ }^{26}$ By the large sample approximation, we have

$$
\begin{aligned}
T= & n_{1}^{-1 / 2} \sum_{l=1}^{L}\left\{F_{l}\left(x_{l\left(i_{l} j_{l}\right)}\right)-1 / 2\right\}+n_{1}^{-1 / 2} \sum_{l=1}^{L}\left\{\hat{F}_{l}\left(x_{l\left(i_{l} j_{l}\right)}\right)\right. \\
& \left.-F_{l}\left(x_{l\left(i_{l} j_{l}\right)}\right)\right\}
\end{aligned}
$$

$$
=n_{1}^{-1 / 2} \sum_{l=1}^{L}\left\{F_{l}\left(x_{l\left(i_{l} j_{l}\right)}\right)-1 / 2\right\}+o_{p}(1),
$$

where $F_{l}\left(x_{l\left(i_{i} j_{l}\right)}\right)-1 / 2$ are mean 0 random variables under $H_{0}$. Let $\varepsilon_{i}=\sum_{(j, l) \in(i)}\left\{F_{l}\left(x_{l\left(i_{j} j_{l}\right)}\right)-1 / 2\right\}$, where $(j, l) \in(i)$ denotes the collection of all eyes $j$ and locations $l$ belonging to patient $i$. Then, by the central limit theorem, under $H_{0}, T$ is approximately normally distributed with mean 0 and variance $\sigma^{2}$ that can be consistently estimated by ${ }^{26}$ :

$$
\hat{\sigma}^{2}=n_{1}^{-1} \sum_{i=1}^{n_{1}} \hat{\varepsilon}_{i}^{2}
$$

where $\hat{\varepsilon}_{i}=\sum_{(j, l) \in(i)}\left\{\hat{F}_{l}\left(x_{l\left(i_{l} j_{l}\right)}\right)-1 / 2\right\}$. Hence, we can reject $H_{0}$ if $|\hat{T} / \hat{\sigma}|>z_{1-\alpha / 2}$, where $z_{1-\alpha / 2}$ is the $100(1-\alpha / 2)$ percentile of the standard normal distribution.

\section{Confidence Intervals}

For location $l$, let $\mu_{l}=E\left(y_{i j l}\right)$ denote the mean PRL thickness for healthy eyes, which is estimated as $\hat{\mu}_{l}=$ $m_{2}^{-1} \sum_{i=1}^{n_{2}} \sum_{j=1}^{m_{2 i}} y_{i j l}$. Then, $\hat{\mu}_{l}$ is approximately normal with mean $\mu_{l}$ and variance that can be estimated by ${ }^{26}$.

$$
\hat{\boldsymbol{\nu}}_{l}=\frac{\sum_{i=1}^{n_{2}}\left\{\sum_{j=1}^{m_{2 i}}\left(y_{i j l}-\hat{\mu}_{l}\right)\right\}^{2}}{m_{2}^{2}}
$$

Hence, a $100(1-\alpha)$ confidence interval for $\mu_{l}$ is given as $\hat{\mu}_{l} \pm z_{1-\alpha / 2} \sqrt{\hat{\nu}_{l}}$.

\section{Appendix 2. Correlation between Change in Photoreceptor Layer Thickness and Drusen Height (or Width)}

For the age-related macular degeneration (AMD) group, let $x_{i j l}$ denote the drusen height (or width) and $\tilde{y}_{i j l}$ denote the photoreceptor layer (PRL) thickness at location $l(=1, \ldots$, $\left.s_{i j}\right)$ for eye $j\left(=1, \ldots, m_{1 i}\right)$ of patient $i\left(=1, \ldots, n_{1}\right)$. Let $\hat{\mu}_{l}$ denote the average PRL thickness at location $l$ from the 17 healthy (control) eyes. For location $l$, the percentage change in the PRL thickness resulting from drusen is measured by $y_{i j l}=100\left(\tilde{y}_{i j l}-\hat{\mu}_{l}\right) / \hat{\mu}_{l}$. We consider a linear regression model

$$
y_{i j l}=\beta_{0}+\beta_{1} x_{i j l}+\varepsilon_{i j l} .
$$

The hypotheses on the association between the drusen height and percentage change in the PRL thickness can be expressed by

$$
H_{0}: \beta_{1}=0 \text { (no association) }
$$

and

$$
H_{1}: \beta_{1} \neq 0 \text { (some association). }
$$

Let $\bar{y}=N^{-1} \sum_{i=1}^{n_{1}} \sum_{j=1}^{m_{1 i}} \sum_{l=1}^{s_{i j}} y_{i j l}$ and $\bar{x}=$ $L_{1}^{-1} \sum_{i=1}^{n_{1}} \sum_{j=1}^{m_{1 i}} \sum_{l=1}^{s_{i j}} x_{i j l}$. By ignoring the dependency among the observation from each patient, the consistent 
estimators of $\left(\beta_{0}, \beta_{1}\right)$ are obtained via regular least square estimator as

$$
\begin{gathered}
\hat{\beta}_{1}=\frac{\sum_{i=1}^{n_{1}} \sum_{j=1}^{m_{1 i}} \sum_{l=1}^{s_{i j}}\left(x_{i j l}-\bar{x}\right) y_{i j l}}{\sum_{i=1}^{n_{1}} \sum_{j=1}^{m_{1 i}} \sum_{l=1}^{s_{i j}}\left(x_{i j l}-\bar{x}\right)^{2}} \\
\hat{\beta}_{0}=\bar{y}-\hat{\beta}_{1} \bar{x} .
\end{gathered}
$$

Considering possible dependency among observations from the 2 eyes of a patient and those from multiple locations of an eye, we obtain the variance estimator of $\hat{\beta}_{1}$ by

$$
\hat{\sigma}^{2}\left(\hat{\beta}_{1}\right)=\frac{\sum_{i=1}^{n_{1}} \hat{\varepsilon}_{i}^{2}}{\left\{\sum_{i=1}^{n_{1}} \sum_{j=1}^{m_{1 i}} \sum_{l=1}^{s_{i j}}\left(x_{i j l}-\bar{x}\right)^{2}\right\}^{2}},
$$

where $\hat{\varepsilon}_{i}=\sum_{j=1}^{m_{1 i}} \sum_{l=1}^{s_{i j}}\left(y_{i j l}-\hat{\beta}_{0}-\hat{\beta}_{1} x_{i j l}\right)\left(x_{i j l}-\bar{x}\right)$. Hence, we reject $H_{0}$ if the absolute value of $T=\hat{\beta}_{1} / \hat{\sigma}\left(\hat{\beta}_{1}\right)$ is large. For large $n_{1}$, the null distribution of $T$ is approximately normal $^{26}$ with mean 0 and variance 1 .

From the data, we obtain $\left(\hat{\beta}_{0}, \hat{\beta}_{1}\right)=(8.865,-0.321)$, $\hat{\sigma}\left(\hat{\beta}_{1}\right)=0.178$, and a 2 -sided $P$ value of 0.0355 using drusen height as the covariate; and $\left(\hat{\beta}_{0}, \hat{\beta}_{1}\right)=(-18.896$, $-0.018), \hat{\sigma}\left(\hat{\beta}_{1}\right)=0.087$, and a 2 -sided $P$ value of 0.4195 using drusen width as the covariate.

\section{Appendix 3. Comparison of the Inner Retina (Photoreceptor Layer, or Retinal Pigment Epithelium) Area in Age-Related Macular Degeneration Eyes versus the Control Eyes}

Let $x_{k i j}$ denote the inner retina (IR; or photoreceptor layer [PRL], retinal pigment epithelium [RPE]) area for eye $j(=$ $\left.1, \ldots m_{k i}\right)$ of subject $i\left(=1, \ldots n_{k}\right)$ from group $k(=1$ for AMD, $=2$ for control). Let $m_{k}=\sum_{i=1}^{n_{k}} m_{k i}$ denote the total number of eyes in group $k$ and $\mu_{k}=E\left(x_{k i j}\right)$ denote the mean IR area for eyes in group $k$ that is estimated as $\bar{x}_{k}=$ $m_{k}^{-1} \sum_{i=1}^{n_{k}} \sum_{j=1}^{m_{k i}} x_{k i j}$.
Note that

$$
\bar{x}_{k}-\mu_{k}=m_{k}^{-1} \sum_{i=1}^{n_{k}} \sum_{j=1}^{m_{k i}}\left(x_{k i j}-\mu_{k}\right)=\sum_{i=1}^{n_{k}} \varepsilon_{i},
$$

where $\left\{\varepsilon_{i}=\sum_{j=1}^{m_{k i}}\left(x_{k i j}-\mu_{k}\right), i=1, \ldots, n_{k}\right\}$ are independent 0 -mean random variables. By the central limit theorem, $\bar{x}_{k}$ is approximately normal with mean $\mu_{k}$ and the variance that can be consistently estimated by

$$
s_{k}^{2}=\frac{\sum_{i=1}^{n_{k}}\left\{\sum_{j=1}^{m_{k i}}\left(x_{k i j}-\bar{x}_{k}\right)\right\}^{2}}{m_{k}^{2}} .
$$

Hence, a $100(1-\alpha)$ confidence interval for $\mu_{k}$ is given as $\bar{x}_{k} \pm z_{1-\alpha / 2} s_{k}$.

To compare the areas between these 2 groups, we test the hypothesis:

$$
H_{0}: \mu_{1}=\mu_{2}
$$

versus

$$
H_{1}: \mu_{1} \neq \mu_{2} \text {. }
$$

Similarly as above, by the central limit theorem, the null distribution of $T=\bar{x}_{1}-\bar{x}_{2}$ is approximated by the normal distribution with mean 0 and variance that can be consistently estimated by

$$
\hat{\sigma}^{2}=\frac{\sum_{i=1}^{n_{1}}\left\{\sum_{j=1}^{m_{1 i}}\left(x_{1 i j}-\bar{x}\right)\right\}^{2}}{m_{1}^{2}}+\frac{\sum_{i=1}^{n_{2}}\left\{\sum_{j=1}^{m_{2 i}}\left(x_{2 i j}-\bar{x}\right)\right\}^{2}}{m_{2}^{2}},
$$

where $\bar{x}=\left(m_{1}+m_{2}\right)^{-1}\left(m_{1} \bar{x}_{1}+m_{2} \bar{x}_{2}\right)$ denotes the pooled sample mean from the eyes of both groups. Hence, we reject $H_{0}$ if $|T / \hat{\sigma}|>z_{1-\alpha / 2}$.

\section{Appendix 4. Correlation among Retinal Pigment Epithelium Area, Inner Retina Area, and Photoreceptor Layer Area}

The sample correlation coefficient $\hat{\rho}$ obtained by ignoring the correlated data is a consistent estimator. As in the regular regression analysis with independent observations, testing on the correlation coefficient $\left(H_{0}: \rho=0\right)$ is identical to the testing on the slope of the linear regression line $\left(H_{0}\right.$ : $\beta_{1}=0$ ), as described in Appendix 2 . 